\title{
Miyaoka-Yau-type inequalities for Kähler-Einstein manifolds
}

\author{
Kwokwai Chan and Naichung Conan Leung
}

\begin{abstract}
We investigate Chern number inequalities on Kähler-Einstein manifolds and their relations to uniformization. For Kähler-Einstein manifolds with $c_{1}>0$, we prove certain Chern number inequalities in the toric case. For Kähler-Einstein manifolds with $c_{1}<0$, we propose a series of Chern number inequalities, interpolating Yau's and Miyaoka's inequalities.
\end{abstract}

\section{Introduction}

Yau's celebrated solution to Calabi's conjecture [17] says that a compact Kähler $n$-fold $X$ with $c_{1}(X)<0$ or $c_{1}(X)=0$ always admit Kähler-Einstein metrics. As an application, he proved in [16] that for the case $c_{1}(X)<0$, we have the following Chern number inequality: ${ }^{1}$

$$
c_{1}^{2}(X)\left|c_{1}(X)\right|^{n-2} \leq \frac{2(n+1)}{n} c_{2}(X)\left|c_{1}(X)\right|^{n-2}
$$

and equality holds if and only if $X$ is uniformized by the unit ball in $\mathbb{C}^{n}$. It turns out that [12] Yau's inequality (1.1) is a consequence of the (Higgs-) stability of the bundle $T_{X}^{*} \oplus \mathcal{O}_{X}$ on $X$.

On the other hand, as a generalization of the Miyaoka-Yau inequality for surfaces [6], ,[16], Miyaoka proved in [7] the following inequality:

$$
c_{1}^{2}(X) H^{n-2} \leq 3 c_{2}(X) H^{n-2}
$$

for any nef class $H$ on a compact Kähler $n$-fold $X$ with $c_{1}(X)<0$. The relation between this inequality and stability also deserves investigation.

\footnotetext{
${ }^{1}$ Throughout this paper, by $\left|c_{1}(X)\right|$, we mean $-c_{1}(X)$ (resp., $\left.c_{1}(X)\right)$ if $c_{1}(X)<0$ (resp., $c_{1}(X)>0$ ).
} 
In 1990, Yau [18] conjectured that the existence of Kähler-Einstein metrics should be equivalent to some notion of stability. This vast program of Yau would reveal deep relations between the existence of Kähler-Einstein metrics, stability, Chern number inequalities and uniformization. However, despite much work done by Yau, Tian, Donaldson and others [14], [18], [13], [3], our understanding of these relations is still poor, especially for the $c_{1}>0$ case.

In view of Yau's program and inequalities (1.1) and (1.2), we propose the following series of Miyaoka-Yau type Chern number inequalities for the $c_{1}<0$ case:

$$
\begin{aligned}
c_{1}^{2}(X)\left|c_{1}(X)\right|^{n-2} & \leq \frac{2(n+1)}{n} c_{2}(X)\left|c_{1}(X)\right|^{n-2} \\
& \vdots \\
c_{1}^{2}(X)\left|c_{1}(X)\right|^{k-2} H^{n-k} & \leq \frac{2(k+1)}{k} c_{2}(X)\left|c_{1}(X)\right|^{k-2} H^{n-k} \\
& \vdots \\
c_{1}(X)^{2} H^{n-2} & \leq 3 c_{2}(X) H^{n-2} .
\end{aligned}
$$

This, in particular, will provide a link between inequalities (1.1) and (1.2). We also infer that an equality will lead to uniformization. More precisely, we propose the following.

Conjecture 1.1. Let $X$ be a compact Kähler $n$-fold with $c_{1}(X)<0$. Then the above series of inequalities $(*)$ hold for any nef class $H$ on $X$. Moreover, equality holds for the kth level inequality with $H^{n-k}$ effective if and only if $X$ is a fibration whose general fiber is uniformized by the $k$-dimensional unit ball and $H$ comes from the base.

In the next section, we will investigate the intimate relation between these inequalities and stability and explain why such a conjecture is conceivable.

For the $c_{1}>0$ case, both the existence of Kähler-Einstein metrics and stability become much more subtle, as mentioned above. Nevertheless, the situation becomes considerably better in the toric case. Recall that for a toric Fano manifold $X$, there is an associated reflexive polytope $P$. Recently, Wang and Zhu [15] proved that a toric Fano manifold admits Kähler-Einstein metrics if and only if it is balanced, i.e., the center of mass of $P$ is at the origin. In view of this, we consider Kähler-Einstein manifolds with $c_{1}>0$ which are toric, and prove, in Sections 3 and Sections 4 , the following Chern number inequalities. 
Theorem 1.2. Let $X$ be a Kähler-Einstein toric Fano $n$-fold. Then for any nef class $H$, we have:

$$
c_{1}^{2}(X) H^{n-2} \leq 3 c_{2}(X) H^{n-2}
$$

provided that either

(i) $n=2,3,4$, or

(ii) each facet of $P$ contains a lattice point in its interior.

Theorem 1.2, together with the discussion at the end of Section 2, yields the following.

Corollary 1.3. Let $X$ be a product of Kähler-Einstein toric Fano manifolds of dimensions less than or equal to 3 . Then the whole set of inequalities (*) hold for any nef class $H$ on $X$.

We will also discuss how the proposed inequalities $(*)$ can give new combinatorial information on balanced reflexive polytopes. Let $P$ be an $n$-dimensional balanced reflexive polytope such that the corresponding toric variety $X$ is smooth. Denote by $\#(k P)$ the number of lattice points in $k P$. The normalized Ehrhart polynomial of $P$ :

$$
\widetilde{E}(P):=\frac{\#(k P)}{\operatorname{Vol}(P)}=b_{n} k^{n}+b_{n-1} k^{n-1}+b_{n-2} k^{n-2}+\cdots
$$

measures the growth of the number of lattice points. Now Yau's inequality gives the following asymptotic lower bound.

Proposition 1.4. For $k$ large, we have

$$
\widetilde{E}(P) \geq \widetilde{E}\left(P_{n}\right)
$$

with equality holds if and only if $P=P_{n}$, where $P_{n}$ denotes $(n+1)$ times of the standard simplex, which is the polytope of $\mathbb{P}^{n}$.

Other inequalities of $(*)$ can give asymptotic upper bounds on Ehrhart polynomials. These will be discussed in the final section.

\section{Manifolds with $c_{1}<0$}

In this section, we first review how Yau's inequality (1.1) can be deduced from stability. Then we explain, along similar lines, how Conjecture 1.1 comes out. 
Let $X$ be a compact Kähler $n$-fold with $c_{1}(X)<0$. The direct sum $E:=T_{X}^{*} \oplus \mathcal{O}_{X}$ of the holomorphic cotangent bundle and the trivial line bundle is of rank $n+1$. Let $\theta \in \Omega^{1,0}(\operatorname{End}(E))$ be the Higgs field given by the identity map $T_{X}^{*} \rightarrow T_{X}^{*}=\mathcal{O}_{X} \otimes T_{X}^{*}$. Then, it can be shown that $(E, \theta)$ is Hermitian-Yang-Mills and hence Higgs-stable with respect to the polarization $\left|c_{1}(X)\right|[12]$. Bogomolov's inequality then gives Yau's inequality:

$$
c_{1}^{2}(X)\left|c_{1}(X)\right|^{n-2} \leq \frac{2(n+1)}{n} c_{2}(X)\left|c_{1}(X)\right|^{n-2}
$$

and equality implies that $X$ is uniformized by the unit ball. In view of this, we suggest that Miyaoka's inequality:

$$
c_{1}^{2}(X) H^{n-2} \leq 3 c_{2}(X) H^{n-2}
$$

also follows from the (Higgs-)stability of some rank-3 bundle, which, analogous to the above situation, should be the direct sum of a rank-2 bundle and a trivial bundle. A natural choice of this is described as follows.

We begin with the rank- $n$ bundle $T_{X}^{*}$ over $X$. Let $\operatorname{Gr}\left(2, T_{X}^{*}\right)$ be the Grassmannian bundle of 2-planes in $T_{X}^{*}, \pi: \operatorname{Gr}\left(2, T_{X}^{*}\right) \rightarrow X$ the projection map. Let $S_{2}$ be the universal rank-2 sub-bundle of $\pi^{*} T_{X}^{*}$. It is natural to expect that Miyaoka's inequality is related to the stability of $S_{2} \oplus \mathcal{O}$. More generally, for $k=3, \ldots, n$, let $\operatorname{Gr}\left(k, T_{X}^{*}\right)$ be the Grassmannian bundle of $k$-planes and $S_{k}$ the universal rank- $k$ bundle. We observe the following relation between the Chern classes of $S_{k}$ and $X$.

\section{Lemma 2.1.}

$$
\begin{aligned}
& c_{1}^{2}(X) c_{1}^{k-2}(X) H^{n-k}=c_{1}^{2}\left(S_{k}\right) c_{1}^{k-2}(X) H^{n-k} \cdot c_{k}^{n-k}\left(S_{k}\right) \\
& c_{2}(X) c_{1}^{k-2}(X) H^{n-k}=c_{2}\left(S_{k}\right) c_{1}^{k-2}(X) H^{n-k} \cdot c_{k}^{n-k}\left(S_{k}\right)
\end{aligned}
$$

where on the right-hand sides, we have suppressed the pull-back.

Proof. Let $Q_{k}:=\pi^{*} T_{X}^{*} / S_{k}$ be the universal quotient bundle. Then $c_{n-r}(X)=$ $c_{n-r-k}\left(Q_{k}\right) c_{k}\left(S_{k}\right)+\cdots+c_{n-r-m}\left(Q_{k}\right) c_{m}\left(S_{k}\right)+\cdots$, for $r=0,1, \ldots, n-k$. Intersecting with $\alpha:=c_{1}^{k-2}(X) H^{n-k}$, all except the first two Chern classes of $X$ become zero. For $r=0, c_{n-k}\left(Q_{k}\right) c_{k}\left(S_{k}\right) \alpha=0$. For $r=1, c_{n-k-1}\left(Q_{k}\right)$ $c_{k}^{2}\left(S_{k}\right) \alpha=-c_{n-k}\left(Q_{k}\right) c_{k}\left(S_{k}\right) c_{k-1}\left(S_{k}\right) \alpha=0$. Recursively, we have

$$
c_{1}\left(Q_{k}\right) c_{k}^{n-k}\left(S_{k}\right) \alpha=0, c_{2}\left(Q_{k}\right) c_{k}^{n-k-1}\left(S_{k}\right) \alpha=0 .
$$

Since $c_{1}\left(Q_{k}\right)=c_{1}(X)-c_{1}\left(S_{k}\right)$, the first equation gives $c_{1}^{2}(X) c_{k}^{n-k}$ $\left(S_{k}\right) \alpha=c_{1}^{2}\left(S_{k}\right) c_{k}^{n-k}\left(S_{k}\right) \alpha$. Then by $c_{2}\left(Q_{k}\right)=c_{2}(X)-c_{2}\left(S_{k}\right)-c_{1}\left(S_{k}\right) c_{1}(X)+$ 
$c_{1}^{2}\left(S_{k}\right), c_{2}(X) c_{k}^{n-k}\left(S_{k}\right) \alpha=c_{2}\left(S_{k}\right) c_{k}^{n-k}\left(S_{k}\right) \alpha$. Finally, if $\widetilde{S}_{k}$ is the universal bundle over the ordinary Grassmannian $\operatorname{Gr}(k, n)$, then the intersection number $c_{k}^{n-k}\left(\widetilde{S}_{k}\right)=1$. This completes the proof of the lemma.

Since $c_{k}^{n-k}\left(\widetilde{S}_{k}\right)=1, \widetilde{X}:=c_{k}^{n-k}\left(S_{k}\right)$ looks like a section of $\operatorname{Gr}\left(k, T_{X}^{*}\right)$ over $X$. Suppose that this is really the case and $\widetilde{X}$ is a genuine manifold. Restrict the bundle $S_{k}$ to $\widetilde{X}$, which we still denote by $S_{k}$. Also denote by $\pi: \widetilde{X} \rightarrow$ $X$ the restriction of the projection map. Consider the direct sum $E_{k}=$ $S_{k} \oplus \mathcal{O}_{\tilde{X}}$. Let $\theta_{k} \in \Omega^{1,0}\left(\operatorname{End}\left(E_{k}\right)\right)$ be the Higgs-field given by the inclusion $S_{k} \hookrightarrow T_{\widetilde{X}}^{*}=\mathcal{O}_{\widetilde{X}} \otimes T_{\widetilde{X}}^{*}$. Suppose that $\left(E_{k}, \theta_{k}\right)$ is Hermitian-Yang-Mills and hence Higgs-stable with respect to the polarization $\pi^{*}\left|c_{1}(X)\right|$. Let $H$ be an ample class on $X$. We can assume that $H$ is very ample by replacing it with a sufficiently high multiple if necessary. Then choosing $n-k$ sections of $\pi^{*} H$ will generically cut out a $k$-dimensional submanifold of $\widetilde{X}$. When restricted to this $k$-dimensional submanifold, $\left(E_{k}, \theta_{k}\right)$ remains Higgs-stable. Therefore, we have

$$
c_{1}^{2}\left(S_{k}\right)\left|c_{1}(X)\right|^{k-2} H^{n-k} \leq \frac{2(k+1)}{k} c_{2}\left(S_{k}\right)\left|c_{1}(X)\right|^{k-2} H^{n-k}
$$

on $\widetilde{X}=c_{k}^{n-k}\left(S_{k}\right)$. By the above lemma, the following inequality holds

$$
c_{1}^{2}(X)\left|c_{1}(X)\right|^{k-2} H^{n-k} \leq \frac{2(k+1)}{k} c_{2}(X)\left|c_{1}(X)\right|^{k-2} H^{n-k} .
$$

Moreover, equality means that any submanifold cut out by a generic choice of $n-k$ sections of $\pi^{*} H$ is uniformized by the $k$-dimensional unit ball. Hence, $\widetilde{X}$ and $X$ should be fibered by these ball-quotients.

Remark 2.2. Of course the above is not a proof! Our aim is to show that Conjecture 1.1 is conceivable and intimately related to stability.

As a test of $(*)$, let us look at $X=M \times N$, a product of manifolds with $c_{1}<0$ of dimensions $m$ and $n$, respectively. Let $H_{1}$ and $H_{2}$ be ample classes on $M$ and $N$, respectively. Then by a direct computation, we have that, for $k=2, \ldots, m+n$, the intersection number

$$
\left(\frac{2(k+1)}{k} c_{2}(X)-c_{1}^{2}(X)\right)\left|c_{1}(X)\right|^{k-2}\left(H_{1}+H_{2}\right)^{m+n-k}
$$


is greater than or equal to the following sum

$$
\begin{aligned}
& \sum_{p=2}^{\min (k, m)} A_{p}\left(\frac{2(p+1)}{p} c_{2}(M)-c_{1}^{2}(M)\right)\left|c_{1}(M)\right|^{p-2} H_{1}^{m-p} \\
& \quad \times\left|c_{1}(N)\right|^{k-p} H_{2}^{n-(k-p)} \\
& \quad+\sum_{p=2}^{\min (k, n)} B_{p}\left(\frac{2(p+1)}{p} c_{2}(N)-c_{1}^{2}(N)\right)\left|c_{1}(N)\right|^{p-2} H_{2}^{n-p} \\
& \quad \times\left|c_{1}(M)\right|^{k-p} H_{1}^{m-(k-p)}
\end{aligned}
$$

where $A_{p}, B_{p}$ are non-negative numbers. It follows that if both $M$ and $N$ satisfy the series of inequalities $(*)$, then the same is true for $X$ (for classes of the form $H_{1}+H_{2}$ ). Moreover, if, say, $M$ is a ball-quotient, then an equality would result for classes $H_{2}$ on $N$, which agrees with our conjecture.

\section{Kähler-Einstein toric Fano manifolds}

Next we turn to Kähler-Einstein manifolds with $c_{1}>0$ which are toric. In this section, we give a proof of:

Theorem 3.1 (=second half of Theorem 1.2). Let $X$ be a KählerEinstein toric Fano $n$-fold, or more generally, toric Fano manifold with reductive automorphism group. Then for any nef class $H$, we have the inequality:

$$
c_{1}^{2}(X) H^{n-2} \leq 3 c_{2}(X) H^{n-2}
$$

provided that each facet of $P$ contains a lattice point in its interior.

We fix our notations first. For details of toric Fano varieties and polytopes, please refer to [1], [4] and [10]. Let $N \cong \mathbb{Z}^{n}$ be a lattice and $M=$ $\operatorname{Hom}_{\mathbb{Z}}(N, \mathbb{Z}) \cong \mathbb{Z}^{n}$ the dual lattice with $\langle\cdot, \cdot\rangle: M \times N \rightarrow \mathbb{Z}$ the natural pairing. Denote by $N_{\mathbb{R}}=N \otimes_{\mathbb{Z}} \mathbb{R} \cong \mathbb{R}^{n}$ and $M_{\mathbb{R}}=M \otimes_{\mathbb{Z}} \mathbb{R} \cong \mathbb{R}^{n}$ (resp., $N_{\mathbb{Q}}$ and $M_{\mathbb{Q}}$ ) the real (resp., rational) scalar extensions. For a subset $S$ in a real vector space, we denote by $\operatorname{conv}(S)$ (resp., aff $(S))$ the convex (resp., affine) hull of $S$.

Let $P \subset M_{\mathbb{R}}$ be a reflexive polytope and $X=X_{P}$ the corresponding toric Fano variety. We assume that $X$ is smooth. Denote by $\mathcal{F}(P)=$ $\left\{F_{1}, F_{2}, \ldots, F_{d}\right\}$ the facets of $P$, and by $D_{1}, D_{2}, \ldots, D_{d}$ the corresponding torus invariant prime divisors on $X$. Then the first and second Chern classes 
are given by:

$$
c_{1}(X)=\sum_{i=1}^{d} D_{i}, \quad c_{2}(X)=\sum_{i<j} D_{i} \cdot D_{j}
$$

We then compute the codimension 2 cycle

$$
3 c_{2}(X)-c_{1}^{2}(X)=3 \sum_{i<j} D_{i} \cdot D_{j}-\left(\sum_{i=1}^{d} D_{i}\right)^{2}=\sum_{i<j} D_{i} \cdot D_{j}-\sum_{i=1}^{d} D_{i}^{2} .
$$

By general theory of toric varieties, each $u \in M_{\mathbb{Q}}$ determines a $\mathbb{Q}$-divisor by:

$$
\operatorname{div}\left(\chi^{u}\right)=\sum_{i=1}^{d}\left\langle u, v_{i}\right\rangle D_{i}
$$

We can move each $D_{i}$ by choosing an element $u_{F_{i}} \in \operatorname{aff}\left(F_{i}\right) \cap M_{\mathbb{Q}}$, so that $\left\langle u_{F_{i}}, v_{i}\right\rangle=-1$, and we have

$$
D_{i}^{2} \sim_{\mathbb{Q}} D_{i} \cdot\left(D_{i}+\sum_{j=1}^{d}\left\langle u_{F_{i}}, v_{j}\right\rangle D_{j}\right)=\sum_{j \neq i}\left\langle u_{F_{i}}, v_{j}\right\rangle D_{i} \cdot D_{j}
$$

where $\sim_{\mathbb{Q}}$ denotes $\mathbb{Q}$-rationally equivalence. Summing up all gives

$$
\sum_{i=1}^{d} D_{i}^{2} \sim_{\mathbb{Q}} \sum_{i=1}^{d} \sum_{j \neq i}\left\langle u_{F_{i}}, v_{j}\right\rangle D_{i} \cdot D_{j}=\sum_{i<j}\left(\left\langle u_{F_{i}}, v_{j}\right\rangle+\left\langle u_{F_{j}}, v_{i}\right\rangle\right) D_{i} \cdot D_{j}
$$

Hence we have

$$
3 c_{2}(X)-c_{1}^{2}(X) \sim_{\mathbb{Q}} \sum_{i<j}\left(1-\left\langle u_{F_{i}}, v_{j}\right\rangle-\left\langle u_{F_{j}}, v_{i}\right\rangle\right) D_{i} \cdot D_{j} .
$$

We formulate the following.

Conjecture 3.2. If the center of mass of $P$, denoted by center $(P)$, is at the origin, then there exists $u_{F_{i}} \in \operatorname{aff}\left(F_{i}\right) \cap M_{\mathbb{Q}}$, for $i=1,2, \ldots, d$ such that

$$
\left\langle u_{F_{i}}, v_{j}\right\rangle \leq \frac{1}{2}
$$

for any adjacent facets $F_{i}$ and $F_{j}$. 
Since this would imply that the cycle class $\left[3 c_{2}(X)-c_{1}^{2}(X)\right]$ is effective, inequality (1.2) for Kähler-Einstein toric Fano manifolds:

$$
\left(3 c_{2}(X)-c_{1}^{2}(X)\right) H^{n-2} \geq 0
$$

for any nef class $H$, would follow. The question is now in purely combinatorial terms.

To prove Theorem 3.1, we recall that a root of a reflexive polytope $P$ is a lattice point contained in the relative interior of some facet. We denote the set of all roots of $P$ by $\mathcal{R}(P)$. $P$ is said to be semisimple if $\mathcal{R}(P)=-\mathcal{R}(P)$. By results of Demazure [10], the automorphism group of $X=X_{P}$, i.e., $\operatorname{Aut}(X)$, is reductive if and only if $P$ is semisimple. While by Matsushima's obstruction to the existence of Kähler-Einstein metrics, if $X$ has KählerEinstein metrics, then $\operatorname{Aut}(X)$ is reductive. Hence, if center $(P)=0$, then $P$ is semisimple. Recently, Nill gave a purely convex-geometrical proof of this in $[9]$.

We will need the following powerful results on the geometry of semisimple reflexive polytopes proved by Nill [9].

Lemma 3.3 (Lemma 2.4. of [9]). Let $P \subset M_{\mathbb{R}}$ be a reflexive polytope. For any $F \in \mathcal{F}(P)$ and $m \in F \cap M$, there is a $\mathbb{Z}$-basis $\left\{e_{1}^{*}, e_{2}^{*}, \ldots, e_{n}^{*}\right\}$ of $M$ such that $m=e_{n}^{*}$ and $F \subset\left\{u \in M_{\mathbb{R}}: u^{n}=\left\langle u, e_{n}\right\rangle=1\right\}$.

Lemma 3.4 (Lemma 5.9. of [9]). Let $P \subset M_{\mathbb{R}}$ be a $n$-dimensional reflexive polytope. Let $m \in \mathcal{R}(P)$ be contained in $F_{m}$ which is associated to the vertex $v_{m} \in \mathcal{V}\left(P^{*}\right)$. Then

1. $P \subset F_{m}-\mathbb{R}_{\geq 0} m, P \cap M \subset(F \cap M)-\mathbb{N} m,\left\{v \in P^{*} \cap N:\langle m, v\rangle<0\right\}$ $=\left\{v_{m}\right\}$.

2. $P=\operatorname{conv}\left(F_{m}, F_{m^{\prime}}\right)$ if and only if there is only one facet $F_{m^{\prime}}$ which is associated to $v_{m^{\prime}} \in \mathcal{V}\left(P^{*}\right)$ with $\left\langle m, v_{m^{\prime}}\right\rangle>0$.

3. $-m \in \mathcal{R}(P)$ if and only if the previous condition is satisfied and $\langle m$, $\left.v_{m^{\prime}}\right\rangle=1$.

In this case $F_{m^{\prime}}=F_{-m}$. Furthermore $F_{m}$ and $F_{-m}$ are naturally isomorphic as lattice polytopes and $\left\{v \in P^{*} \cap N:\langle m, v\rangle \neq 0\right\}=\left\{v_{m}, v_{-m}\right\}$.

Now Theorem 3.1 is an immediate consequence of the following lemma.

Lemma 3.5. Suppose that a facet, say $F_{1} \in \mathcal{F}(P)$, contains a root $m \in$ $\mathcal{R}(P)$ such that $-m \in \mathcal{R}(P)$. Then there exists $u_{F_{1}} \in$ aff $\left(F_{1}\right) \cap M_{\mathbb{Q}}$ such that

$$
\left\langle u_{F_{1}}, v_{j}\right\rangle \leq \frac{1}{2}
$$


for any facet $F_{j}$ adjacent to $F_{1}$.

Proof. Since $-m \in \mathcal{R}(P)$, by Lemma 3.4 , there is a unique facet, say $F_{2} \in$ $\mathcal{F}(P)$ such that $\left\langle m, v_{2}\right\rangle=1$ and

$$
\left\{v \in P^{*} \cap N:\langle m, v\rangle \neq 0\right\}=\left\{v_{1}, v_{2}\right\} .
$$

Suppose that $F_{2}$ is not adjacent to $F_{1}$. Then we can simply take $u_{F_{1}}=m$ and we have

$$
\left\langle u_{F_{1}}, v_{j}\right\rangle=0<\frac{1}{2}
$$

for any $F_{j}$ adjacent to $F_{1}$.

If $F_{2}$ is adjacent to $F_{1}$, then we choose a $\mathbb{Z}$-basis $\left\{e_{1}^{*}, e_{2}^{*}, \ldots, e_{n}^{*}\right\}$ of $M$ such that $m=e_{n}^{*}$ and $F \subset\left\{u \in M_{\mathbb{R}}: u^{n}=\left\langle u, e_{n}\right\rangle=1\right\}$ by Lemma 3.3. In particular, we have $v_{1}=-e_{n}$. Suppose $F_{2}, F_{3}, \ldots, F_{l}$ are all the facets adjacent to $F_{1}$. By Lemma 3.4, $P=\operatorname{conv}\left(F_{1}, F_{2}\right)$. We observe that $v_{2}=z_{2}+e_{n}$ and $v_{j}=z_{j}$ for $j=3, \ldots, l$, where $z_{2}, z_{3}, \ldots, z_{l}$ are elements in the span of $\left\{e_{1}, \ldots, e_{n-1}\right\}$. Regard $F_{1}$ as a polytope in the affine hyperplane $H_{1}=\{u \in$ $\left.M_{\mathbb{R}}: u^{n}=\left\langle u, e_{n}\right\rangle=1\right\}$ with respect to the lattice aff $\left(F_{1}\right) \cap M$ and with origin $m$. Using the coordinates with respect to the basis $\left\{e_{1}^{*}, \ldots, e_{n-1}^{*}\right\}, F_{1}$ is given by

$$
F_{1}=\left\{y \in H_{1}:\left\langle y, z_{2}\right\rangle \geq-2,\left\langle y, z_{j}\right\rangle \geq-1 \text { for } j=3, \ldots, l\right\} .
$$

Also we have

$$
\begin{aligned}
& F_{1} \cap F_{2} \subset\left\{y \in H_{1}:\left\langle y, z_{2}\right\rangle=-2\right\} \quad \text { and } \\
& F_{1} \cap F_{j} \subset\left\{y \in H_{1}:\left\langle y, z_{j}\right\rangle=-1\right\}
\end{aligned}
$$

for $j=3, \ldots, l$. Consider the polytope $Q \subset F_{1}$ defined by

$$
Q=\left\{y \in F_{1}:\left\langle y, z_{2}\right\rangle \leq 1,\left\langle y, z_{j}\right\rangle \leq \frac{1}{2} \text { for } j=3, \ldots, l\right\} .
$$

In fact, $Q=(-1 / 2) F_{1}$. $Q$ is not empty since the origin (i.e., $m$ ) is contained in it. Moreover, there is a positive constant $\epsilon>0$ such that the ball

$$
B_{\epsilon}=\left\{y \in H_{1}:|y| \leq \epsilon\right\}
$$

is contained in $Q$. We claim that there exists $y \in Q$ such that

$$
\left\langle y, z_{2}\right\rangle \leq-\frac{1}{2}
$$


Suppose not, then any vertex of $Q$, say $w$, will satisfy

$$
\left\langle w, z_{2}\right\rangle>-\frac{1}{2}
$$

Since $Q=(-1 / 2) F_{1}$ and $F_{1}$ is integral, this implies that

$$
\left\langle w, z_{2}\right\rangle \geq 0
$$

But $Q$ is the convex hull of its vertices, so we have

$$
\left\langle y, z_{2}\right\rangle \geq 0
$$

for all $y \in Q$. This is a contradiction since $B_{\epsilon}$ contains points with $\left\langle y, z_{2}\right\rangle<0$. This proves our claim. It follows that there exists $y \in Q$, with rational coordinates such that

$$
\left\langle y, z_{2}\right\rangle \leq-\frac{1}{2} \quad \text { and } \quad\left\langle y, z_{j}\right\rangle \leq \frac{1}{2} \text { for } j=3, \ldots, l .
$$

If we set $u_{F_{1}}=y+e_{n}$, then this is equivalent to

$$
\left\langle u_{F_{1}}, v_{j}\right\rangle \leq \frac{1}{2}
$$

for $j=2,3, \ldots, l$.

Remark 3.6. The difficulty in removing the last condition in Theorem 3.1 can be described as follows. For a facet not containing a root, there are examples, such as the $\mathbb{P}^{1}$-bundle $\mathbb{P}\left(\mathcal{O}_{\mathbb{P}^{1} \times \mathbb{P}^{1}} \otimes \mathcal{O}_{\mathbb{P}^{1} \times \mathbb{P}^{1}}(1,-1)\right)$, such that the possible values of $u_{F_{i}} \in \operatorname{aff}\left(F_{i}\right) \cap M_{\mathbb{Q}}$, though form a sub-polytope of $F_{i}$, is of dimension less than that of $F_{i}$, in other words, the sub-polytope has empty relative interior. It is then more difficult to locate the required $u_{F_{i}}$. Indeed, we do not see why such a sub-polytope is always non-empty.

\section{Further evidence}

In this section, we will prove:

Theorem 4.1 (=first half of Theorem 1.2). Let $X$ be a KählerEinstein toric Fano surface, 3-fold or 4-fold. Then we have

$$
c_{1}^{2}(X) H^{n-2} \leq 3 c_{2}(X) H^{n-2}
$$

for any nef class $H$. 
Since Kähler-Einstein toric $n$-folds for $n \leq 4$ are completely classified, we can and will prove Theorem 4.1 by directly checking the inequality for all cases along the lines of Conjecture 3.2.

For any complex surface, it is well known that the Miyaoka-Yau inequality $c_{1}^{2} \leq 3 c_{2}$ is true, so we are done. In fact there are exactly three KählerEinstein toric Fano surfaces, namely,

$$
\mathbb{P}^{2}, \quad \mathbb{P}^{1} \times \mathbb{P}^{1}, \quad S_{3}
$$

where $S_{3}$ is blow-up of $\mathrm{P}^{2}$ at three points, which is a del Pezzo surface of degree 6 . It is easy to check the inequality for them directly.

For 3 -folds and 4-folds, we need a lemma:

Lemma 4.2. Let $X=Y \times Z$ be a product of varieties. Suppose $\left[3 c_{2}(Y)-\right.$ $\left.c_{1}^{2}(Y)\right]$ and $\left[3 c_{2}(Z)-c_{1}^{2}(Z)\right]$ are effective (resp., positive) cycle classes on $Y$ and $Z$, respectively. Then $\left[3 c_{2}(X)-c_{1}^{2}(X)\right]$ is also effective (resp., positive).

Proof. Note that effectivity and positivity of a cycle class is preserved under pull-back via a projection. Now the lemma follows from $3 c_{2}(X)-c_{1}^{2}(X)=$ $\left(3 c_{2}(Z)-c_{1}^{2}(Z)\right)+c_{1}(Y) c_{1}(Z)$ if $\operatorname{dim}(Y)=1$ and $\operatorname{dim}(Z) \geq 2$, and from $3 c_{2}(X)-c_{1}^{2}(X)=\left(3 c_{2}(Y)-c_{1}^{2}(Y)\right)+\left(3 c_{2}(Z)-c_{1}^{2}(Z)\right)+c_{1}(Y) c_{1}(Z)$ if both $\operatorname{dim}(Y), \operatorname{dim}(Z) \geq 2$. Here we have suppressed the pull-backs.

Kähler-Einstein toric Fano 3-folds are classified by Mabuchi [5]. There are five examples. The first four are $\mathbb{P}^{3}$ and products of lower-dimensional smooth toric Fano varieties:

$$
\mathbb{P}^{2} \times \mathbb{P}^{1}, \quad \mathbb{P}^{1} \times \mathbb{P}^{1} \times \mathbb{P}^{1}, \quad \mathbb{P}^{1} \times S_{3}
$$

By the above lemma, Conjecture 3.2 is true for these four examples. The remaining one is the $\mathbb{P}^{1}$-bundle

$$
\mathbb{P}\left(\mathcal{O}_{\mathbb{P}^{1} \times \mathbb{P}^{1}} \otimes \mathcal{O}_{\mathbb{P}^{1} \times \mathbb{P}^{1}}(1,-1)\right)
$$

The verification for this will be carried out in the appendix.

Kähler-Einstein toric Fano 4-folds are classified by Nakagawa and Batyrev and Selivanova $[8,2]$. There are 12 of them. The first nine are $\mathbb{P}^{4}$ and the 
following eight products are of lower-dimensional varieties:

$$
\begin{gathered}
\mathbb{P}^{1} \times \mathbb{P}^{3}, \quad \mathbb{P}^{1} \times \mathbb{P}^{1} \times \mathbb{P}^{2}, \\
\mathbb{P}^{2} \times \mathbb{P}^{2}, \quad \mathbb{P}^{1} \times \mathbb{P}^{1} \times \mathbb{P}^{1} \times \mathbb{P}^{1}, \\
\mathbb{P}^{2} \times S_{3}, \quad \mathbb{P}^{1} \times \mathbb{P}^{1} \times S_{3}, \quad S_{3} \times S_{3}, \\
\mathbb{P}^{1} \times \mathbb{P}\left(\mathcal{O}_{\mathbb{P}^{1} \times \mathbb{P}^{1}} \otimes \mathcal{O}_{\mathbb{P}^{1} \times \mathbb{P}^{1}}(1,-1)\right) .
\end{gathered}
$$

Applying Lemma 4.2 again, Conjecture 3.2 holds for these examples. The remaining three are, in the notations of [2], $V_{2}, W_{2}$ and $X_{1,1}$. We again check the inequality for them in the appendix. This completes the proof of Theorem 4.1.

Remark 4.3. There is a notion of symmetric toric Fano manifolds introduced by Batyrev-Selivanova in [2]. Namely, the toric Fano manifold $X$ associated to a polytope $P$, is called symmetric if the $\operatorname{group}^{\operatorname{Aut}_{M}(P) \text { of }}$ lattice automorphisms leaving $P$ invariant has only the origin as a fix point. As noticed by Batyrev-Selivanova, all known examples of Kähler-Einstein toric Fano manifolds (including all examples of dimensions 2, 3 and 4) are symmetric. It is not known whether the inequality (1.2) is true for symmetric toric Fano manifolds.

\section{Applications to counting lattice points}

In this section, we apply our results to give new asymptotic bounds on counting lattice points of balanced reflexive polytopes.

Let $X=X_{P}$ be a toric Fano variety associated to a reflexive polytope $P \subset \mathbb{R}^{n}$. Let $H$ be an ample divisor on $X$ and $P_{H} \subset \mathbb{R}^{n}$ be the corresponding polytope. Suppose that $X$ is smooth, then $k H$ is very ample for every $k \geq 1$. The higher cohomology groups of the line bundle $\mathcal{O}(k H)$ vanish and the sections precisely correspond to the lattice points in $k P_{H}$. Hence, by the Riemann-Roch the Ehrhart polynomial [4, Section 5.3]:

$$
\begin{aligned}
E\left(P_{H}\right) & =\#\left(k P_{H}\right)=\sum_{\nu=0}^{n} a_{\nu} k^{\nu} \\
& =a_{n} k^{n}+a_{n-1} k^{n-1}+a_{n-2} k^{n-2}+O\left(k^{n-3}\right)
\end{aligned}
$$

where $a_{\nu}=\frac{1}{\nu !}\left(H^{\nu} \cdot \operatorname{Td}_{\nu}(X)\right)$ and $\operatorname{Td}_{\nu}(X)$ denotes the $\nu$ th Todd class of $X$. The first few Todd classes are given by

$$
\operatorname{Td}_{n}(X)=1, \quad \operatorname{Td}_{n-1}(X)=\frac{1}{2} c_{1}(X), \quad \operatorname{Td}_{n-2}(X)=\frac{1}{12}\left(c_{1}^{2}(X)+c_{2}(X)\right) .
$$


Hence we have

$$
a_{n}=\frac{H^{n}}{n !}=\operatorname{Vol}\left(P_{H}\right), \quad a_{n-1}=\frac{1}{2} \frac{H^{n-1} c_{1}(X)}{(n-1) !}=\frac{1}{2} \operatorname{Vol}\left(\partial P_{H}\right)
$$

Since $A_{*}(X)_{\mathbb{Q}}$ is spanned by the classes $[V(\tau)], \tau \in \Delta_{P}$, the $\nu$ th Todd class can be written as

$$
\operatorname{Td}_{\nu}(X)=\sum_{\tau \in \Delta_{P}(\nu)} r_{\tau}[V(\tau)]
$$

for some rational numbers $r_{\tau}$. A famous question raised by Danilov is that given a lattice $M$ (or $N$ ), whether we can choose the $r_{\tau}$ 's uniformly, independent of the toric variety $X$. Of course, by the above, we have uniformly, $r_{\tau}=1 / 2$, for all $\tau \in \Delta_{P}(1)$. There has been much work on this question [11].

Applying inequality (1.2), we can get a uniform bound on $r_{\tau}$ for $\tau \in$ $\Delta_{P}(2)$. Namely, if inequality (1.2) is true for $X$, then we have

$$
\operatorname{Td}_{n-2}(X) H^{n-2}=\frac{1}{12}\left(c_{1}^{2}(X)+c_{2}(X)\right) H^{n-2} \leq \frac{1}{3} c_{2}(X) H^{n-2}
$$

from which follows that

$$
r_{\tau} \leq \frac{1}{3}
$$

for any $\tau \in \Delta_{P}(2)$. Also, for the coefficient $a_{n-2}$ in the above formula for the number of lattice points, we have

$$
a_{n-2}=\frac{1}{(n-2) !}\left(H^{n-2} \cdot \operatorname{Td}_{n-2}(X)\right) \leq \frac{1}{3} \frac{H^{n-2} c_{2}(X)}{(n-2) !} .
$$

The quantity $H^{n-2} c_{2}(X) /(n-2)$ ! is the sum of volumes of codimension 2 faces of $P_{H}$. If we denote by $P_{H}(2)$ the union of codimension 2 faces of $P_{H}$, then we get the following asymptotic upper bound for the growth of number of lattice points of $k P_{H}$ :

$$
E\left(k P_{H}\right) \leq \operatorname{Vol}\left(P_{H}\right) k^{n}+\frac{1}{2} \operatorname{Vol}\left(\partial P_{H}\right) k^{n-1}+\frac{1}{3} \operatorname{Vol}\left(P_{H}(2)\right) k^{n-2}+O\left(k^{n-3}\right)
$$

provided that inequality (1.2) holds for $X$. Hence by the results of previous sections, we have 
Proposition 5.1. If $P$ is balanced, then the following asymptotic upper bound holds:

$$
E\left(k P_{H}\right) \leq \operatorname{Vol}\left(P_{H}\right) k^{n}+\frac{1}{2} \operatorname{Vol}\left(\partial P_{H}\right) k^{n-1}+\frac{1}{3} \operatorname{Vol}\left(P_{H}(2)\right) k^{n-2}+O\left(k^{n-3}\right)
$$

provided that either

(i) $n=2,3,4$, or

(ii) each facet of $P$ contains a root.

On the other hand, taking $H$ to be the anticanonical divisor $K^{-1}=$ $c_{1}(X)$, then the normalized Ehrhart polynomial of $P$ is given by

$$
\widetilde{E}(P):=\frac{\#(k P)}{\operatorname{Vol}(P)}=b_{n} k^{n}+b_{n-1} k^{n-1}+b_{n-2} k^{n-2}+\cdots
$$

where in general, we have $b_{n}=1, b_{n-1}=1 / 2 n$ and

$$
b_{n-2}=\frac{a_{n-2}}{\operatorname{Vol}(P)}=\frac{1}{12(n-2) ! \operatorname{Vol}(P)}\left(c_{1}^{2}(X)+c_{2}(X)\right) c_{1}^{n-2}(X) .
$$

By Yau's inequality (1.1), we have

Proposition 5.2 (= Proposition 1.4). If $P$ is balanced, then for $k$ large, we have

$$
\widetilde{E}(P) \geq \widetilde{E}\left(P_{n}\right)
$$

with equality holds if and only if $P=P_{n}$, where $P_{n}$ is the polytope of $\mathbb{P}^{n}$.

\section{Appendix A}

\section{A.1 The last four examples}

In this appendix, we verify Conjecture 3.2 for the remaining low dimension examples. In the following, $\left\{e_{1}, e_{2}, \ldots, e_{n}\right\}$ will always denote the standard basis of the lattice $N \cong \mathbb{Z}^{n}$ and $P$ the corresponding polytope. Recall that we have to prove that there exists $u_{F_{i}} \in \operatorname{aff}\left(F_{i}\right) \cap M_{\mathbb{Q}}$, for $i=1,2, \ldots, d$ such that

$$
\left\langle u_{F_{i}}, v_{j}\right\rangle \leq \frac{1}{2}
$$

for any adjacent facets $F_{i}$ and $F_{j}$. 
For $\mathbb{P}\left(\mathcal{O}_{\mathbb{P}^{1} \times \mathbb{P}^{1}} \otimes \mathcal{O}_{\mathbb{P}^{1} \times \mathbb{P}^{1}}(1,-1)\right)$, the six vertices of $P^{*}$ are given by

$$
\mathcal{V}\left(P^{*}\right)=\left\{e_{1}, e_{2}, e_{3},-e_{3},-e_{1}-e_{3},-e_{2}+e_{3}\right\}
$$

which correspond to the six facets of $P$ :

$$
\begin{array}{ll}
F_{1}=\operatorname{conv}\left(u_{1}, u_{2}, u_{3}, u_{4}\right), & F_{2}=\operatorname{conv}\left(u_{1}, u_{2}, u_{5}, u_{6}\right), \\
F_{3}=\operatorname{conv}\left(u_{1}, u_{3}, u_{5}, u_{7}\right), & F_{4}=\operatorname{conv}\left(u_{2}, u_{4}, u_{6}, u_{8}\right), \\
F_{5}=\operatorname{conv}\left(u_{5}, u_{6}, u_{7}, u_{8}\right), & F_{6}=\operatorname{conv}\left(u_{3}, u_{4}, u_{7}, u_{8}\right),
\end{array}
$$

where $u_{1}, u_{2}, \ldots, u_{8}$ are vertices of $P$ given, with respect to the dual basis $\left\{e_{1}^{*}, e_{2}^{*}, e_{3}^{*}\right\}$ of $M$, by the column vectors of the following matrix

$$
\left(\begin{array}{cccccccc}
-1 & -1 & -1 & -1 & 2 & 0 & 2 & 0 \\
-1 & -1 & 0 & 2 & -1 & -1 & 0 & 2 \\
-1 & 1 & -1 & 1 & -1 & 1 & -1 & 1
\end{array}\right)
$$

respectively. We can choose $u_{F_{1}}, u_{F_{2}}, u_{F_{3}}, u_{F_{4}}, u_{F_{5}}$ and $u_{F_{6}}$ to be the column vectors of

$$
\left(\begin{array}{rrrrrr}
-1 & 0 & \frac{1}{2} & -\frac{1}{2} & 1 & 0 \\
0 & -1 & -\frac{1}{2} & \frac{1}{2} & 0 & 1 \\
0 & 0 & -1 & 1 & 0 & 0
\end{array}\right)
$$

respectively.

$V_{2}$ is the so-called del Pezzo variety of dimension 4, which is centrally symmetric. The 10 vertices of $P^{*}$ are given by

$$
\begin{aligned}
\mathcal{V}\left(P^{*}\right)= & \left\{e_{1}, e_{2}, e_{3}, e_{4},-\left(e_{1}+e_{2}+e_{3}+e_{4}\right),\right. \\
& \left.-e_{1},-e_{2},-e_{3},-e_{4}, e_{1}+e_{2}+e_{3}+e_{4}\right\}
\end{aligned}
$$

which correspond to the 10 facets of $P$ :

$$
\begin{aligned}
& F_{1}=\operatorname{conv}\left(u_{1}, u_{2}, u_{3}, u_{4}, u_{5}, u_{6}, u_{7}, u_{8}, u_{9}, u_{10}, u_{11}, u_{12}\right), \\
& F_{2}=\operatorname{conv}\left(u_{1}, u_{2}, u_{3}, u_{13}, u_{14}, u_{15}, u_{16}, u_{17}, u_{18}, u_{19}, u_{20}, u_{21}\right), \\
& F_{3}=\operatorname{conv}\left(u_{4}, u_{5}, u_{6}, u_{13}, u_{14}, u_{15}, u_{22}, u_{23}, u_{24}, u_{25}, u_{26}, u_{27}\right), \\
& F_{4}=\operatorname{conv}\left(u_{7}, u_{8}, u_{9}, u_{16}, u_{17}, u_{18}, u_{22}, u_{23}, u_{24}, u_{28}, u_{29}, u_{30}\right),
\end{aligned}
$$




$$
\begin{aligned}
F_{5} & =\operatorname{conv}\left(u_{10}, u_{11}, u_{12}, u_{19}, u_{20}, u_{21}, u_{25}, u_{26}, u_{27}, u_{28}, u_{29}, u_{30}\right) \\
F_{6} & =\operatorname{conv}\left(u_{13}, u_{14}, u_{16}, u_{17}, u_{19}, u_{20}, u_{22}, u_{23}, u_{25}, u_{26}, u_{28}, u_{29}\right) \\
F_{7} & =\operatorname{conv}\left(u_{4}, u_{5}, u_{7}, u_{8}, u_{10}, u_{11}, u_{22}, u_{24}, u_{25}, u_{27}, u_{28}, u_{30}\right) \\
F_{8} & =\operatorname{conv}\left(u_{1}, u_{2}, u_{7}, u_{9}, u_{10}, u_{12}, u_{16}, u_{18}, u_{19}, u_{21}, u_{29}, u_{30}\right) \\
F_{9} & =\operatorname{conv}\left(u_{1}, u_{3}, u_{4}, u_{6}, u_{11}, u_{12}, u_{13}, u_{15}, u_{20}, u_{21}, u_{26}, u_{27}\right) \\
F_{10} & =\operatorname{conv}\left(u_{2}, u_{3}, u_{5}, u_{6}, u_{8}, u_{9}, u_{14}, u_{15}, u_{17}, u_{18}, u_{23}, u_{24}\right)
\end{aligned}
$$

where $u_{1}, u_{2}, \ldots, u_{30}$ are the 30 vertices of $P$. The first one-third is given, with respect to the dual basis $\left\{e_{1}^{*}, e_{2}^{*}, e_{3}^{*}, e_{4}^{*}\right\}$ of $M$, by the column vectors of the following matrix

$$
\left(\begin{array}{cccccccccc}
-1 & -1 & -1 & -1 & -1 & -1 & -1 & -1 & -1 & -1 \\
-1 & -1 & -1 & 1 & 1 & 0 & 1 & 1 & 0 & 1 \\
1 & 1 & 0 & -1 & -1 & -1 & 1 & 0 & 1 & 1 \\
1 & 0 & 1 & 1 & 0 & 1 & -1 & -1 & -1 & 0
\end{array}\right)
$$

respectively, the second one-third is given by the columns of

$$
\left(\begin{array}{cccccccccc}
-1 & -1 & 1 & 1 & 0 & 1 & 1 & 0 & 1 & 1 \\
1 & 0 & -1 & -1 & -1 & -1 & -1 & -1 & -1 & -1 \\
0 & 1 & -1 & -1 & -1 & 1 & 0 & 1 & 1 & 0 \\
1 & 1 & 1 & 0 & 1 & -1 & -1 & -1 & 0 & 1
\end{array}\right)
$$

respectively, and the last one-third is given by the columns of

$$
\left(\begin{array}{cccccccccc}
0 & 1 & 1 & 0 & 1 & 1 & 0 & 1 & 1 & 0 \\
-1 & 1 & 0 & 1 & 1 & 0 & 1 & 1 & 0 & 1 \\
1 & -1 & -1 & -1 & -1 & -1 & -1 & 0 & 1 & 1 \\
1 & -1 & -1 & -1 & 0 & 1 & 1 & -1 & -1 & -1
\end{array}\right)
$$

respectively. We can choose $u_{F_{i}} \in \operatorname{aff}\left(F_{i}\right) \cap M_{\mathbb{Q}}, i=1,2, \ldots, 10$, to be given by the column vectors of the matrix

$$
\left(\begin{array}{cccccccccc}
-1 & \frac{1}{2} & \frac{1}{2} & \frac{1}{2} & \frac{1}{2} & 1 & -\frac{1}{2} & -\frac{1}{2} & -\frac{1}{2} & -\frac{1}{2} \\
\frac{1}{2} & -1 & \frac{1}{2} & \frac{1}{2} & \frac{1}{2} & -\frac{1}{2} & 1 & -\frac{1}{2} & -\frac{1}{2} & -\frac{1}{2} \\
\frac{1}{2} & \frac{1}{2} & -1 & 0 & 0 & -\frac{1}{2} & -\frac{1}{2} & 1 & 0 & 0 \\
0 & 0 & 0 & -1 & 0 & 0 & 0 & 0 & 1 & 0
\end{array}\right),
$$

respectively. 
Geometrically, $W_{2}$ is the blown-up of $\mathbb{P}^{2} \times \mathbb{P}^{2}$ along 3 codimension 2 subvarieties $Z_{i} \cong \mathbb{P}^{1} \times \mathbb{P}^{1}$ defined by the equations $z_{i}=0, z_{i}^{\prime}=0, i=0,1,2$. Combinatorially, the vertices of $P^{*}$ are given by the following nine vectors

$$
\begin{aligned}
\mathcal{V}\left(P^{*}\right)= & \left\{e_{1}, e_{2}, e_{3}, e_{4},-e_{1}-e_{2},-e_{3}-e_{4},\right. \\
& \left.-e_{1}-e_{2}-e_{3}-e_{4}, e_{1}+e_{3}, e_{2}+e_{4}\right\}
\end{aligned}
$$

which correspond to the 9 facets of $P$ :

$$
\begin{aligned}
& F_{1}=\operatorname{conv}\left(u_{1}, u_{2}, u_{3}, u_{4}, u_{5}, u_{6}, u_{7}, u_{8}, u_{9}, u_{10}\right) \\
& F_{2}=\operatorname{conv}\left(u_{1}, u_{2}, u_{3}, u_{11}, u_{12}, u_{13}, u_{14}, u_{15}, u_{16}, u_{17}\right) \\
& F_{3}=\operatorname{conv}\left(u_{11}, u_{12}, u_{13}, u_{14}, u_{15}, u_{18}, u_{19}, u_{20}, u_{21}, u_{22}\right) \\
& F_{4}=\operatorname{conv}\left(u_{4}, u_{5}, u_{6}, u_{7}, u_{8}, u_{18}, u_{19}, u_{20}, u_{23}, u_{24}\right) \\
& F_{5}=\operatorname{conv}\left(u_{4}, u_{5}, u_{9}, u_{11}, u_{12}, u_{16}, u_{18}, u_{19}, u_{21}, u_{23}\right) \\
& F_{6}=\operatorname{conv}\left(u_{1}, u_{2}, u_{6}, u_{7}, u_{10}, u_{13}, u_{14}, u_{17}, u_{22}, u_{24}\right) \\
& F_{8}=\operatorname{conv}\left(u_{1}, u_{3}, u_{5}, u_{8}, u_{9}, u_{10}, u_{14}, u_{15}, u_{18}, u_{20}, u_{21}, u_{22}\right) \\
& F_{9}=\operatorname{conv}\left(u_{2}, u_{3}, u_{7}, u_{8}, u_{12}, u_{15}, u_{16}, u_{17}, u_{19}, u_{20}, u_{23}, u_{24}\right),
\end{aligned}
$$

where $u_{1}, u_{2}, \ldots, u_{24}$ are the 24 vertices of $P$. The first half are given, with respect to the dual basis $\left\{e_{1}^{*}, e_{2}^{*}, e_{3}^{*}, e_{4}^{*}\right\}$ of $M$, by the column vectors of the following matrix

$$
\left(\begin{array}{cccccccccccc}
-1 & -1 & -1 & -1 & -1 & -1 & -1 & -1 & -1 & -1 & 2 & 2 \\
-1 & -1 & -1 & 2 & 2 & 1 & 0 & 0 & 2 & 1 & -1 & -1 \\
0 & 1 & 0 & 1 & 0 & 2 & 2 & 0 & 0 & 0 & -1 & -1 \\
1 & 0 & 0 & -1 & -1 & -1 & -1 & -1 & 0 & 1 & 1 & 0
\end{array}\right)
$$

respectively, while the second half are given by the columns of

$$
\left(\begin{array}{cccccccccccc}
1 & 0 & 0 & 2 & 1 & 0 & 1 & 0 & 0 & 0 & 1 & 0 \\
-1 & -1 & -1 & -1 & -1 & 1 & 0 & 0 & 1 & 0 & 0 & 0 \\
-1 & -1 & -1 & 0 & 1 & -1 & -1 & -1 & -1 & -1 & 1 & 2 \\
2 & 2 & 0 & 0 & 0 & -1 & -1 & -1 & 1 & 2 & -1 & -1
\end{array}\right)
$$


respectively. We can choose $u_{F_{i}} \in \operatorname{aff}\left(F_{i}\right) \cap M_{\mathbb{Q}}, i=1,2, \ldots, 9$, to be given by the column vectors of the matrix

$$
\left(\begin{array}{ccccccccc}
-1 & \frac{1}{2} & \frac{3}{5} & -\frac{3}{10} & \frac{1}{2} & -\frac{3}{10} & \frac{1}{4} & -\frac{1}{2} & \frac{1}{4} \\
\frac{1}{2} & -1 & -\frac{3}{10} & \frac{3}{5} & \frac{1}{2} & -\frac{3}{10} & \frac{1}{4} & \frac{1}{4} & -\frac{1}{2} \\
\frac{3}{5} & -\frac{3}{10} & -1 & \frac{1}{2} & -\frac{3}{10} & \frac{1}{2} & \frac{1}{4} & -\frac{1}{2} & \frac{1}{4} \\
-\frac{3}{10} & \frac{3}{5} & \frac{1}{2} & -1 & -\frac{3}{10} & \frac{1}{2} & \frac{1}{4} & \frac{1}{4} & -\frac{1}{2}
\end{array}\right)
$$

respectively.

Finally for $X_{1,1}$, the 10 vertices of $P^{*}$ are given by

$$
\mathcal{V}\left(P^{*}\right)=\left\{e_{1}, e_{2}, e_{3},-e_{3}, e_{4},-e_{4}, e_{3}-e_{4},-e_{3}+e_{4},-e_{1}+e_{3},-e_{2}-e_{3}\right\}
$$

which correspond to the 10 facets of $P$ :

$$
\begin{aligned}
F_{1} & =\operatorname{conv}\left(u_{1}, u_{2}, u_{3}, u_{4}, u_{5}, u_{6}, u_{7}, u_{8}, u_{9}, u_{10}, u_{11}, u_{12}\right) \\
F_{2} & =\operatorname{conv}\left(u_{1}, u_{2}, u_{3}, u_{4}, u_{5}, u_{6}, u_{13}, u_{14}, u_{15}, u_{16}, u_{17}, u_{18}\right) \\
F_{3} & =\operatorname{conv}\left(u_{1}, u_{2}, u_{7}, u_{8}, u_{13}, u_{14}, u_{19}, u_{20}\right) \\
F_{4} & =\operatorname{conv}\left(u_{3}, u_{4}, u_{9}, u_{10}, u_{15}, u_{16}, u_{21}, u_{22}\right) \\
F_{5} & =\operatorname{conv}\left(u_{1}, u_{5}, u_{7}, u_{11}, u_{13}, u_{17}, u_{19}, u_{23}\right) \\
F_{6} & =\operatorname{conv}\left(u_{3}, u_{6}, u_{9}, u_{12}, u_{15}, u_{18}, u_{21}, u_{24}\right) \\
F_{7} & =\operatorname{conv}\left(u_{2}, u_{6}, u_{8}, u_{12}, u_{14}, u_{18}, u_{20}, u_{24}\right) \\
F_{8} & =\operatorname{conv}\left(u_{4}, u_{5}, u_{10}, u_{11}, u_{16}, u_{17}, u_{22}, u_{23}\right) \\
F_{9} & =\operatorname{conv}\left(u_{13}, u_{14}, u_{15}, u_{16}, u_{17}, u_{18}, u_{19}, u_{20}, u_{21}, u_{22}, u_{23}, u_{24}\right) \\
F_{10} & =\operatorname{conv}\left(u_{7}, u_{8}, u_{9}, u_{10}, u_{11}, u_{12}, u_{19}, u_{20}, u_{21}, u_{22}, u_{23}, u_{24}\right)
\end{aligned}
$$

where $u_{1}, u_{2}, \ldots, u_{24}$ are the 24 vertices of $P$. The first half is given, with respect to the dual basis $\left\{e_{1}^{*}, e_{2}^{*}, e_{3}^{*}, e_{4}^{*}\right\}$ of $M$, by the column vectors of the following matrix

$$
\left(\begin{array}{cccccccccccc}
-1 & -1 & -1 & -1 & -1 & -1 & -1 & -1 & -1 & -1 & -1 & -1 \\
-1 & -1 & -1 & -1 & -1 & -1 & 2 & 2 & 0 & 0 & 1 & 1 \\
-1 & -1 & 1 & 1 & 0 & 0 & -1 & -1 & 1 & 1 & 0 & 0 \\
-1 & 0 & 1 & 0 & -1 & 1 & -1 & 0 & 1 & 0 & -1 & 1
\end{array}\right)
$$


respectively, while the second half is given by the columns of

$$
\left(\begin{array}{cccccccccccc}
0 & 0 & 2 & 2 & 1 & 1 & 0 & 0 & 2 & 2 & 1 & 1 \\
-1 & -1 & -1 & -1 & -1 & -1 & 2 & 2 & 0 & 0 & 1 & 1 \\
-1 & -1 & 1 & 1 & 0 & 0 & -1 & -1 & 1 & 1 & 0 & 0 \\
-1 & 0 & 1 & 0 & -1 & 1 & -1 & 0 & 1 & 0 & -1 & 1
\end{array}\right)
$$

respectively. We can choose $u_{F_{i}} \in \operatorname{aff}\left(F_{i}\right) \cap M_{\mathbb{Q}}, i=1,2, \ldots, 10$, to be given by the column vectors of the matrix

$$
\left(\begin{array}{cccccccccc}
-1 & 0 & -\frac{1}{2} & \frac{1}{2} & -\frac{1}{4} & \frac{1}{4} & -\frac{1}{4} & \frac{1}{4} & 1 & 0 \\
0 & -1 & \frac{1}{2} & -\frac{1}{2} & \frac{1}{4} & -\frac{1}{4} & \frac{1}{4} & -\frac{1}{4} & 0 & 1 \\
0 & 0 & -1 & 1 & -\frac{1}{2} & \frac{1}{2} & -\frac{1}{2} & \frac{1}{2} & 0 & 0 \\
0 & 0 & -\frac{1}{2} & \frac{1}{2} & -1 & 1 & \frac{1}{2} & -\frac{1}{2} & 0 & 0
\end{array}\right)
$$

respectively.

\section{Acknowledgements}

We thank the referee for many helpful comments and pointing out an earlier mistake. Both authors are partially supported by RGC grants from the Hong Kong Government and the second author is also partially supported by CUHK Direct Grant 2060275.

\section{References}

[1] V. Batyrev, Dual polyhedra and mirror symmetry for Calabi-Yau hypersurfaces in toric varieties, J. Algebraic Geom. 3 (1994), no. 3, 493-535.

[2] V. Batyrev and E. Selivanova, Einstein-Kähler metrics on symmetric toric Fano manifolds, J. Reine Angew. Math. 512 (1999), $225-236$.

[3] S. Donaldson, Scalar curvature and stability of toric varieties, J. Diff. Geom. 62 (2002), no. 2, 289-349.

[4] W. Fulton, Introduction to toric varieties, Annals of Mathematics Studies, 131, Princeton University Press, Princeton, 1993. 
[5] T. Mabuchi, Einstein-Kähler forms, Futaki invariants and convex geometry on toric Fano varieties, Osaka J. Math. 24 (1987), no. 4, 705-737.

[6] Y. Miyaoka, On the Chern numbers of surfaces of general type, Invent. Math. 42 (1977), 225-237.

[7] Y. Miyaoka, The Chern classes and Kodaira dimension of a minimal variety, Algebraic Geometry (Sendai, 1985), 449-476, Adv. Stud. Pure Math., 10, North-Holland, Amsterdam, 1987.

[8] Y. Nakagawa, Einstein-Kähler toric Fano fourfolds, Tôhoku Math. J. (2) 45 (1993), no. 2, 297-310.

[9] B. Nill, Complete toric varieties with reductive automorphism group, Math. Z. 252 (2006), no. 4, 767-786.

[10] T. Oda, Convex bodies and algebraic geometry, Ergebnisse der Mathematik und ihrer Grenzgebiete (3), 15, Springer-Verlag, Berlin, 1988 .

[11] J. Pommersheim and H. Thomas, Cycles representating the Todd class of a toric variety, J. Amer. Math. Soc. 17 (2004), no. 4, 983-994.

[12] C. Simpson, Constructing variations of Hodge structure using YangMills theory and applications to uniformization, J. Amer. Math. Soc. 1 (1988), no. 4, 867-918.

[13] G. Tian, Kähler-Einstein metrics with positive scalar curvature, Invent. Math. 130 (1997), no. 1, 1-37.

[14] G. Tian and S.-T. Yau, Kähler-Einstein metrics on complex surfaces with $C_{1}>0$, Comm. Math. Phys. 112 (1987), no. 1, 175-203.

[15] X.-J. Wang and X. Zhu, Kähler-Ricci solitons on toric manifolds with positive first Chern class, Adv. Math. 188 (2004), no. 1, 87-103.

[16] S.-T. Yau, Calabi's conjecture and some new results in algebraic geometry, Proc. Natl. Acad. Sci. USA. 74 (1977), no. 5, 1798-1799.

[17] S.-T. Yau, On the Ricci curvature of a compact Kähler manifold and the complex Monge-Ampère equation I, Comm. Pure Appl. Math. 31 (1978), no. 3, 339-411. 
[18] S.-T. Yau, Open problems in geometry, Differential Geometry: Partial Differential Equations on Manifolds (Los Angeles, CA, 1990), 1-28, Proc. Sympos. Pure Math., 54, Part 1, American Mathematical Society, Providence, RI, 1993.

Department of Mathematics

The Institute of Mathematical Sciences

The Chinese University of Hong Kong

SHATIN

HONG KONG

E-mail address: kwchan@math.cuhk.edu.hk

E-mail address: leung@ims.cuhk.edu.hk

Received February 3, 2006 
